\title{
REPRESENTATIONS OF SEMISIMPLE LIE GROUPS. III
}

\author{
BY

\section{HARISH-CHANDRA}

The main object of this paper is to define the character of an irreducible quasi-simple $\left(^{1}\right)$ representation $\pi$ of a connected semisimple Lie group $G$ on a Hilbert space $\mathfrak{S}$. This will be done as follows. Let $C_{c}^{\infty}(G)$ be the class of all functions on $G$ which are indefinitely differentiable and which vanish outside a compact set. For any $f \in C_{c}^{\infty}(G)$ we consider the operator $\int f(x) \pi(x) d x$ where $d x$ is the Haar measure on $G$. It turns out that this operator has a trace (which we denote by $\left.T_{\pi}(f)\right)$ and the mapping $T_{\pi}: f \rightarrow T_{\pi}(f)\left(f \in C_{c}^{\infty}(G)\right)$ is a distribution in the sense of L. Schwartz [9] such that $T_{\pi}\left(f_{a}\right)=T_{\pi}(f)$ where $f_{a}(x)$ $=f\left(a x a^{-1}\right)(a, x \in G)$. This distribution is defined to be the character of $\pi$. We shall see that two such representations $\pi_{1}, \pi_{2}$ are infinitesimally equivalent (see $[6, \S 9]$ ) if and only if they have the same character. Therefore in particular a unitary irreducible representation is determined within unitary equivalence by its character (cf. Theorem 8 of [6]).

In the last section we give a simple proof of a formula for "spherical functions" on a complex semisimple group. This formula was obtained by Gelfand and Naimark $[1 ; 2]$ in some special cases by direct computation.

1. Some preliminary results. We keep to the notation of our two earlier papers $[6,7]$ on the same subject. $G$ is a connected, simply connected, semisimple Lie group and $g_{0}$ is its Lie algebra over the field $R$ of real numbers. $\mathfrak{g}$ is the complexification of $\mathfrak{g}_{0}$ and $\mathfrak{k}, \mathfrak{p}, \mathfrak{f}_{0}, \mathfrak{p}_{0}, \mathfrak{c}, \mathfrak{f}^{\prime}$, and $\mathfrak{m}$ are defined as in $[6, \S 2]$ and $[7, \S 2] . K, K^{\prime}$, and $D$ are the analytic subgroups of $G$ corresponding to $\mathfrak{f}_{0}, \mathfrak{f}_{0}^{\prime}=\mathfrak{f}^{\prime} \cap \mathfrak{g}_{0}$ and $\mathfrak{c}_{0}=\mathfrak{c} \cap \mathfrak{g}_{0}$ respectively. Let $Z$ be the center of $G$ and $\mathcal{Z}$ the center of the enveloping algebra $\mathfrak{B}$ of $\mathfrak{g}$. If $\pi$ is a representation of $G$ on a Banach space we shall say that $\pi$ is quasi-simple if it maps the elements of $D \cap Z$ and $Z$ into scalar multiples of the unit operator (see $[6, \S 10]$ ).

Let $\pi$ be a quasi-simple irreducible representation of $G$ on a Banach space $\mathfrak{S}$. We denote by $\Omega$ the set of all equivalence classes of finite-dimensional simple representations of $K$. Let $\mathfrak{W}_{\mathfrak{D}}(\mathfrak{D} \in \Omega)$ denote the set of all elements in $\mathfrak{S}$ which transform under $\pi(K)$ according to $\mathfrak{D}$. We know (see [6, Lemma 33]) that $\operatorname{dim} \mathfrak{S}_{\mathfrak{D}}<\infty$. Let $E_{\mathfrak{D}}$ denote the canonical projection of $\mathfrak{S C D}_{\mathfrak{D}}$ on $\mathfrak{S}_{\mathfrak{D}}$ (see $[6, \S 9])$. For any $x \in G$ consider the operator $E_{\mathfrak{D}} \pi(x) E_{\mathfrak{D}}$. It maps $\mathfrak{S}_{\mathfrak{D}}$ into itself and $\mathfrak{S}_{\mathfrak{D}}$, into $\{0\}\left(\mathfrak{D}^{\prime} \neq \mathfrak{D}\right)$. Let $\mathrm{sp}\left(E_{\mathfrak{D}} \pi(x) E_{\mathfrak{D}}\right)$ denote the trace of the restriction of $E_{\mathfrak{D}} \pi(x) E_{\mathfrak{D}}$ on $\mathfrak{S}_{\mathfrak{D}}$. Since $\operatorname{dim} \mathfrak{S}_{\mathfrak{D}}<\infty$ this trace is well defined. Now any given linear function $\alpha$ on $\mathfrak{S}_{\mathfrak{D}}$ may be extended to a continuous linear function on $\mathfrak{S}$ by setting $\alpha(\psi)=\alpha\left(E_{\mathfrak{D}} \psi\right)(\psi \in \mathfrak{S})$. In particular if $\psi_{i}$,

Received by the editors January 9, 1953.

(1) See $[6, \S 10]$ for the definition of a quasi-simple representation. 
$1 \leqq i \leqq r$, is a base for $\mathfrak{S}_{\mathbb{D}}$ and $\widetilde{\psi}_{i}$ is the linear function on $\mathfrak{S}_{\mathfrak{D}}$ which takes the value 1 at $\psi_{i}$ and zero at $\psi_{j}(j \neq i, 1 \leqq i, j \leqq r)$ we may extend $\widetilde{\psi}_{i}$ on $\mathfrak{S}$ in the above fashion. Then it is clear that

$$
\phi_{\mathfrak{D}}^{\pi}(x)=\operatorname{sp} E_{\mathfrak{D} \pi}(x) E_{\mathfrak{D}}=\sum_{i=1}^{r}\left(\tilde{\psi}_{i}, \pi(x) \psi_{i}\right)
$$

in the notation of $[6, \S 10]$. Hence it follows from Lemmas 19 and 34 of [6] that $\phi_{\mathfrak{D}}^{\pi}$ is an analytic function on $G . X_{1}, \cdots, X_{n}$ being a base for $\mathrm{g}_{0}$ over $R$, set $X(t)=t_{1} X_{1}+\cdots+t_{n} X_{n}\left(t_{j} \in R\right)$. Then we know (cf. Theorem 2 and Lemma 34 of [6]) that if $|t|=\max _{j}\left|t_{j}\right|$ is sufficiently small we get the convergent expansions

$$
\pi(\exp X(t)) \psi_{i}=\sum_{m \geqq 0} \frac{1}{m !} \pi\left((X(t))^{m}\right) \psi_{i}, \quad 1 \leqq i \leqq r .
$$

From this it follows immediately that if $z$ is any element in $\mathfrak{B}$ the value of $\sum_{1 \leqq i \leqq r}\left(\tilde{\psi}_{i}, \pi(z) \psi_{i}\right)$ can be obtained in terms of the various partial derivatives of $\phi_{\mathfrak{D}}^{\pi}(\exp X(t))$ with respect to $(t)$ at $t_{1}=t_{2}=\cdots=t_{n}=0$. Let $\sigma$ be the representation of $\mathfrak{A}=\mathfrak{O X}$ (see $[7, \S 2]$ for notation) on $\mathfrak{S}_{\mathfrak{D}}$ defined under $\pi$. Then the knowledge of the function $\phi_{\mathfrak{D}}^{\pi}$ determines in particular $\operatorname{sp} \sigma(z)$ for any $z \in \mathfrak{A}$. Now we know (see Theorem 5 of [6]) that $\pi$ defines a quasi-simple( ${ }^{2}$ ) irreducible representation of $\mathfrak{B}$ on $\mathfrak{S}^{(0)}=\sum_{\mathcal{D}^{\prime} \in \Omega} \mathfrak{S}_{\mathfrak{D}^{\prime}}$ and therefore $\sigma$ is irreducible (see Corollary 2 to Theorem 2 of [7]). On the other hand a finite-dimensional simple representation of an associative algebra is completely determined within equivalence by its trace (see Lemma 16 of [7]). Hence in view of Theorem 2 of [7] we can conclude that the function $\phi_{\mathfrak{D}}^{\pi}$ determines the representation of $\mathfrak{B}$ on $\mathfrak{S}^{(0)}$ up to equivalence and therefore the representation $\pi$ of $G$ up to infinitesimal equivalence. This result may be stated in a slightly more general form as follows.

TheOREM( $\left(^{3}\right)$ 1. Let $\pi_{1}, \cdots, \pi_{r}$ be a finite set of quasi-simple irreducible representations of $G$ on Banach spaces. Suppose no two of them are infinitesimally equivalent. Then all the nonzero functions in the set $\phi_{\mathfrak{D}_{1}^{1}}^{\pi_{1}}, \cdots, \phi_{\mathfrak{D}_{r}}^{\pi_{r}}\left(\mathfrak{D}_{i} \in \Omega\right.$, $1 \leqq i \leqq r)$ are linearly independent.

Let $C$ be the field of complex numbers. If our assertion is false we may suppose that $c_{1} \phi_{\mathfrak{D}_{1}}^{\pi_{1}}+\cdots+c_{s} \phi_{\mathfrak{D}_{s}}^{\pi_{s}}=0$ where $c_{j} \phi_{\mathfrak{D}_{j}}^{\pi_{j}} \neq 0,1 \leqq j \leqq s \quad\left(c_{j} \in C\right)$. Let $\mathfrak{S}_{i}$ be the representation space of $\pi_{i}$. Consider the representation $\sigma_{i}$ of $\mathfrak{A}$ on $\mathfrak{S}_{i}, \mathfrak{D}_{i}(1 \leqq i \leqq s)$ induced under $\pi_{i}$. Then $c_{1} \operatorname{sp} \sigma_{1}(a)+\cdots+c_{s} \operatorname{sp} \sigma_{s}(a)=0$ for all $a \in \mathfrak{A}$. Since $c_{j} \phi_{\mathfrak{D}_{j}^{j}}^{\pi_{j}} \neq 0, \mathfrak{D}_{j}$ occurs in $\pi_{j}$. Moreover $\pi_{j}, \pi_{k}(j \neq k)$ are not infinitesimally equivalent $(1 \leqq j, k \leqq s)$. Hence it follows from Corollary 2 to Theorem 2 of [7] that the representations $\sigma_{1}, \cdots, \sigma_{s}$ are irreducible and no

(2) See [7, end of \$2] for the definition of quasi-simplicity in this case.

(3) Cf. Theorem 7 of $[8(a)]$ and Theorem 2 of $[8(b)]$. 
two of them are equivalent. This however gives a contradiction with Lemma 16 of [7]. So the theorem is proved.

We recall that for two irreducible unitary representations on Hilbert spaces the notions of infinitesimal equivalence and ordinary equivalence are the same (see Theorem 8 of [6]). Hence if $\pi_{1}, \pi_{2}$ are two such representations which are not equivalent, the corresponding functions $\phi_{\mathbb{D}_{1}}^{\pi_{1}}, \phi_{\mathfrak{D}_{2}}^{\pi_{2}}$ are always distinct unless they are both zero.

Theorem 5 of [7] can now be rephrased in terms of the function $\phi_{\mathfrak{D}}^{\pi}$ as follows:

THEOREM $\left({ }^{4}\right)$ 2. Let $\pi$ be a quasi-simple irreducible representation of $G$ on a Banach space $\mathfrak{S}$. Suppose $\mathfrak{D}_{0}$ is a class in $\Omega$ occurring in $\pi$ such that $d\left(\mathfrak{D}_{0}\right)=1$. Then $\operatorname{dim} \mathfrak{S E}_{\mathfrak{D}_{0}}=1$ and it is possible to choose linear functions $\Lambda$ and $\mu$ on $\mathfrak{h}$ and $\mathfrak{c}$ respectively such that

$$
\phi_{D_{0}}^{\boldsymbol{x}}(x)=\int_{K^{*}} e^{\mu\left(\Gamma\left(x, u^{*}\right)\right)} e^{\Lambda\left(H\left(x, u^{*}\right)\right)} d u^{*} \quad(x \in G)
$$

and the infinitesimal character of $\pi$ is $\chi_{\Delta}$.

Some properties of the function $\phi_{\mathfrak{D}}^{\pi}$ have been studied by R. Godement $[3]$ (see also $[1 ; 2]$ ).

We shall now state a few immediate consequences of the results proved in [6].

TheOREM(5) 3. Let $\chi$ be a homomorphism of $\mathbb{3}$ into $C$ and $\mathfrak{D}_{0}$ a class in $\Omega$. Then apart from infinitesimal equivalence there exist only a finite number of irreducible quasi-simple representations $\pi$ of $G$ which have the infinitesimal character $\chi$ and such that $\mathfrak{D}_{0}$ occurs in $\pi$.

This follows from Theorem 2 of [7]. Similarly the following result is obtained from Theorem 3 of [8].

TheOREM 4. Let $\pi$ be a quasi-simple irreducible representation of $G$ on a Banach space $\mathfrak{S}$. Then there exists an integer $N$ such that

$$
\operatorname{dim} \mathfrak{S C D}_{\mathfrak{D}} \leqq N(d(\mathfrak{D}))^{2}
$$

for all $\mathfrak{D} \in \Omega$.

2. Trace of an operator. Let $\left\{c_{\alpha}\right\}_{\alpha \in J}$ be an indexed set of complex numbers. We define the convergence of the series $\sum_{\alpha \in J} c_{\alpha}$ and its sum in the usual manner (see $\S 5$ of [6]). Let $A$ be a bounded operator on a Hilbert space $\mathfrak{S}$ and let $\left\{\psi_{\alpha}\right\}_{\alpha \in J}$ be an orthonormal base for $\mathfrak{S}$. We say that $A$ has a trace

(4) Cf. Theorem 3 of [8(b)]. Our notation is the same as that of Theorem 5 of [7].

(5) Cf. Theorem 6 of $[8(a)]$. 
(or $A$ is of the trace class) if for every such base the series( $\left(^{6}\right) \sum_{\alpha \in J}\left(\psi_{\alpha}, A \psi_{\alpha}\right)$ converges to a sum which is independent of the choice of the base. The value of this sum is called the trace of $A$ and we shall denote it by sp $A$.

Lemma 1. Let $\left\{\psi_{\alpha}\right\}_{\alpha \in J}$ be an orthonormal base for a Hilbert space $\mathfrak{S}$ and $T$ a bounded operator such that $\sum_{\alpha, \beta \in J}\left|t_{\alpha \beta}\right|<\infty$ where $t_{\alpha \beta}=\left(\psi_{\alpha}, T \psi_{\beta}\right)$. Then if $A$ and $B$ are any bounded operators on $\mathfrak{E}, A T B, B A T, T B A$ are all of the trace class and

$$
\text { sp }(A T B)=\operatorname{sp}(B A T)=\operatorname{sp}(T B A) \text {. }
$$

Put $a_{\alpha \beta}=\left(\psi_{\alpha}, A \psi_{\beta}\right), b_{\alpha \beta}=\left(\psi_{\alpha}, B \psi_{\beta}\right) \quad(\alpha, \beta \in J)$ and consider the series $\sum_{\alpha, \beta, \gamma \in J}\left|a_{\alpha \beta} t_{\beta \gamma} b_{\gamma \alpha}\right|$. Then $\left(^{7}\right)$

$$
\begin{aligned}
\sum_{\alpha}\left|a_{\alpha \beta} t_{\beta \gamma} b_{\gamma \alpha}\right| & =\left|t_{\beta \gamma}\right| \sum_{\alpha}\left|a_{\alpha \beta} b_{\gamma \alpha}\right| \\
& \leqq\left|t_{\beta \gamma}\right|\left(\sum_{\alpha}\left|a_{\alpha \beta}\right|^{2}\right)^{1 / 2}\left(\sum_{\alpha}\left|b_{\gamma \alpha}\right|^{2}\right)^{1 / 2} \leqq\left|t_{\beta \gamma}\right||A||B|
\end{aligned}
$$

since

$$
\sum_{\alpha}\left|a_{\alpha \beta}\right|^{2}=\sum_{\alpha}\left|\left(\psi_{\alpha}, A \psi_{\beta}\right)\right|^{2}=\left|A \psi_{\beta}\right|^{2} \leqq|A|^{2}
$$

and similarly for $B$. Hence

$$
\sum_{\alpha, \beta, \gamma}\left|a_{\alpha \beta} t_{\beta \gamma} b_{\gamma \alpha}\right| \leqq|A||B| \sum_{\beta, \gamma}\left|t_{\beta \gamma}\right|<\infty .
$$

This proves that the series $\sum_{\alpha, \beta, \gamma} a_{\alpha \beta} t_{\beta \gamma} b_{\gamma \alpha}$ is absolutely convergent and so it follows in the usual way that

$$
\sum_{\alpha}\left(\psi_{\alpha}, A T B \psi_{\alpha}\right)=\sum_{\alpha}\left(\psi_{\alpha}, T B A \psi_{\alpha}\right)=\sum_{\alpha}\left(\psi_{\alpha}, B A T \psi_{\alpha}\right) .
$$

Now let $U$ be a unitary transformation on $\mathfrak{B}$. Consider $U^{-1} A T B U$. Since $U^{-1} A$ and $B U$ are bounded operators, we can conclude from the above result that

$$
\begin{aligned}
\sum_{\alpha}\left(U \psi_{\alpha}, A T B U \psi_{\alpha}\right) & =\sum_{\alpha}\left(\psi_{\alpha}, U^{-1} A T B U \psi_{\alpha}\right) \\
& =\sum_{\alpha}\left(\psi_{\alpha}, T B U U^{-1} A \psi_{\alpha}\right)=\sum_{\alpha}\left(\psi_{\alpha}, T B A \psi_{\alpha}\right) \\
& =\sum_{\alpha}\left(\psi_{\alpha}, A T B \psi_{\alpha}\right) .
\end{aligned}
$$

Since every orthonormal base in $\mathfrak{S}$ is related to the base $\left\{\psi_{\alpha}\right\}_{\alpha \in J}$ by a unitary transformation, this proves that $A T B$ is of the trace class. Since $B A$ is a

$\left({ }^{(}\right)$As usual we denote by $(\phi, \psi)$ the scalar product of $\phi$ and $\psi$ in $\mathfrak{b}$.

(7) For any bounded operator $Q$ we put $|Q|=\sup _{|\psi|} \leqq 1|Q \psi|$. 
bounded operator it follows from this result that $B A T$ and $T B A$ are also of the trace class. Hence in view of the above equalities we conclude that sp $A T B=\operatorname{sp} B A T=\operatorname{sp} T B A$.

Corollary. If $T$ satisfies the conditions of the above lemma and if $A$ is a regular operator, then $T$ and $A T A^{-1}$ are both of the trace class and $\mathrm{sp} A T A^{-1}$ $=\operatorname{sp} T$.

3. An auxiliary lemma. In order to prove that certain given operators are of the trace class we shall frequently need the following result.

Lemma 2. Let $\mathfrak{l}$ be a semisimple Lie algebra over $C$ of rank $l$. Then the series $\sum_{\mathbb{D}} d(\mathfrak{D})^{-(l+1)}$ is convergent. Here $\mathfrak{D}$ runs over all equivalence classes of finitedimensional simple representations of $\mathfrak{l}$ and $d(\mathfrak{D})$ is the degree of any representation in $\mathfrak{D}$.

Let $\mathfrak{h}$ be a Cartan subalgebra of $\mathfrak{l}$. Choose a fundamental system of roots and let $\Lambda_{1}, \cdots, \Lambda_{l}$ be a fundamental set of dominant integral functions on $\mathfrak{h}$ with respect to this system (see $[5$, Part I]). Then every such function can be written as $m_{1} \Lambda_{1}+\cdots+m_{l} \Lambda_{l}$ where $m_{i}$ are all nonnegative integers. Let $H_{1}, \cdots, H_{l}$ be a base for $\mathfrak{h}$. Extend this to a base $X_{1}, \cdots, X_{n}(n \geqq l)$ for $\mathfrak{l}$ so that $X_{i}=H_{i}, 1 \leqq i \leqq l$. Put $g_{i j}=\operatorname{sp}\left(\operatorname{ad} X_{i}\right.$ ad $\left.X_{j}\right), 1 \leqq i, j \leqq n$, where $X$ $\rightarrow \operatorname{ad} X$ is the adjoint representation of $\mathfrak{l}$. Since $\mathfrak{l}$ is semisimple the matrix $\left(g_{i j}\right)_{1 \leqq i, j \leqq n}$ is nonsingular. Let $\left(g^{i j}\right)_{1 \leqq i, j \leqq n}$ denote its inverse. Let $\mathfrak{U}$ be the enveloping algebra of $\mathfrak{l}$. Put $\omega=\sum_{1 \leqq i, j \leqq n} g^{i j} X_{i} X_{j} \in \mathfrak{U}$. $\omega$ is called the Casimir operator of $\mathfrak{l}$ and it is well known that $\omega$ lies in the center of $\mathfrak{u}$. For any dominant integral function $\Lambda$ on $\mathfrak{h}$ put

$$
|\Lambda|^{2}=\sum_{1 \leqq i, j \leqq l} g^{i j} \Lambda\left(H_{i}\right) \Lambda\left(H_{j}\right) .
$$

Then it is known (see for example [4]) that $|\Lambda|^{2}$ is a positive real number unless $\Lambda=0$. Now let $\sigma$ be an irreducible finite-dimensional representation of $\mathfrak{U}$ and let $\mathfrak{D}$ be the class of $\sigma$. We denote by $\Lambda_{\mathfrak{D}}$ the highest weight of $\sigma$ and by $\omega_{D}$ the number such that $\sigma(\omega)=\omega_{D} \sigma(1)$. Then it follows from Lemma 6 of [4] that $\omega_{D}$ is real, $\omega_{D} \geqq\left|\Lambda_{D}\right|^{2}$, and there exists a real number $\kappa$ such that $\kappa d(\mathfrak{D})^{2} \geqq \omega_{\mathfrak{D}}$ for every irreducible class $\mathfrak{D}$. Hence

$$
d(\mathfrak{D})^{-1} \leqq \kappa^{1 / 2}\left|\Lambda_{\mathscr{D}}\right|^{-1} .
$$

Now the base $H_{1}, \cdots, H_{l}$ can be so chosen that every root of $\mathfrak{l}$ takes real values at $H_{1}, \cdots, H_{l}$. For such a base the quadratic form $\sum_{i, j=1}^{l} g^{i j} x_{i} \cdot x_{j}$ $\left(x_{i} \in R\right)$ is real and positive definite. We can therefore select $H_{1}, \cdots, H_{l}$ in such a way that this form reduces to $x_{1}^{2}+\cdots+x_{l}^{2}$. For any dominant integral function $\Lambda$ let $e_{\Lambda}$ denote the vector in the $l$-dimensional real Euclidean space with the components $\Lambda\left(H_{i}\right)$. Then the set of all points $e_{\Delta}$ form one "octant" of a lattice whose generators are $e_{i}=e_{\Lambda_{i}}, 1 \leqq i \leqq l$. Now 


$$
\sum_{\mathscr{D}}^{\prime} d(\mathfrak{D})^{-(l+1)} \leqq \kappa^{(l+1) / 2} \sum_{\mathfrak{D}}^{\prime}\left|\Lambda_{\mathbb{D}}\right|^{-(l+1)}
$$

where $\sum_{\mathscr{D}}^{\prime}$ denotes the sum over all irreducible classes $\mathfrak{D}$ except the one corresponding to the zero representation of degree 1 . Since each class is completely determined by its highest weight, it follows that

$$
\sum_{D}^{\prime}\left|\Lambda_{D}\right|^{-(l+1)} \leqq \sum_{(m) \geq_{0}}\left|m_{1} e_{1}+\cdots+m_{l} e_{l}\right|^{-(l+1)}
$$

where $|e|$ is the Euclidean length of the vector $e$ and $\sum_{(m) \geqq 0}^{\prime}$ denotes summation over all sets of nonnegative integers $\left(m_{1}, \cdots, m_{l}\right)$ such that $m_{1}+\cdots+m_{l}>0$. Since the series on the right is well known to be convergent, the lemma follows.

4. A result on convergence. We use the terminology of $[6, \S 9]$. Let $\pi$ be a permissible representation of $G$ on a Banach space $\mathfrak{S}$ and $E_{\mathfrak{D}}$ the canonical projection of $\mathfrak{W}$ on the space $\mathfrak{S C D}_{\mathfrak{D}}$ consisting of all elements in $\mathfrak{E}$ which transform under $\pi(K)$ according to $\mathfrak{D}(\mathfrak{D} \in \Omega)$. We shall now prove the following lemma $\left({ }^{8}\right)$.

Lemma 3. There exists an element $z \in \mathfrak{X}$ such that

$$
\sum_{\mathfrak{D} \in \Omega}\left|E_{\mathcal{D}} \psi\right| \leqq|\pi(z) \psi|
$$

for any differentiable element $\psi$ in $\mathfrak{S}$. Moreover the series

$$
\sum_{D \in p} E_{D} \psi
$$

converges to $\psi$.

Let $u \rightarrow u^{*}(u \in K)$ denote the natural mapping of $K$ on $K^{*}=K / D \cap Z$. For any $u \in K$ we denote by $\Gamma(u)$ the unique element in $\mathfrak{c}_{0}$ such that $u \exp (-\Gamma(u)) \in K^{\prime}$. Choose a base $\Gamma_{1}, \cdots, \Gamma_{r}$ for $\mathfrak{c}_{0}$ over $R$ such that $\exp \Gamma_{i}$, $1 \leqq i \leqq r$, is a set of generators for $D \cap Z$. Let $\mu$ be a linear function on $\mathrm{c}$ such that $\pi\left(\exp \Gamma_{i}\right)=e^{\mu\left(\Gamma_{i}\right)} \pi(1), 1 \leqq i \leqq r$. Let $\Omega_{\pi}$ be the set of all classes in $\Omega$ which occur in $\pi$. Then it is clear that if $\mathfrak{D} \in \Omega_{\pi}$ and $\sigma$ is any representation in $\mathfrak{D}$, we must have

$$
\sigma\left(\Gamma_{i}\right)=\left(2 \pi(-1)^{1 / 2} n_{i}+\mu\left(\Gamma_{i}\right)\right) \sigma(1)
$$

where $n_{i}, 1 \leqq i \leqq r$, are all integers. Define a linear function $n_{D}$ on $c$ by setting $n_{\mathcal{D}}\left(\Gamma_{i}\right)=n_{i}, 1 \leqq i \leqq r$, and put $\left|n_{\mathbb{D}}\right|=\left(1+n_{1}^{2}+\cdots+n_{r}^{2}\right)^{1 / 2}$. Then if $w=1$ $-\left(1 / 4 \pi^{2}\right) \sum_{i=1}^{r}\left(\Gamma_{i}-\mu\left(\Gamma_{i}\right)\right)^{2} \in \mathfrak{X}, \sigma(w)=\left|n_{\mathcal{D}}\right|^{2} \sigma(1)$. We note that $w$ lies in the center of $\mathfrak{X}$. Let $\mathfrak{X}^{\prime}$ be the subalgebra of $\mathfrak{B}$ generated by $\left(1, \mathfrak{l}^{\prime}\right)$. Since $\mathfrak{f}^{\prime}$ is semisimple we can find (see Lemma 4 of [7]) an element $z_{0}$ in the center of $\mathfrak{X}^{\prime}$ such that $\sigma\left(z_{0}\right)=d_{\sigma}^{2} \sigma(1)$ for any simple representation $\sigma$ of $\mathfrak{X}$ of degree $d_{\sigma}$.

Put $\pi^{*}\left(u^{*}\right)=e^{-\mu(\Gamma(u))} \pi(u)(u \in K)$. Then $\pi^{*}$ is a representation of $K^{*}$ on

(8) Cf. Lemma 31 of [6] which was stated without proof. 
$\mathfrak{S}$ and if $\mathfrak{D} \in \Omega_{\pi}$

$$
E_{\mathfrak{D}}=d(\mathfrak{D}) \int_{K^{*}} \operatorname{conj}\left(\xi \mathfrak{D}\left(u^{*}\right)\right) \pi^{*}\left(u^{*}\right) d u^{*}\left({ }^{9}\right)
$$

where $\xi_{\infty}$ is the character (on $K^{*}$ ) of the class according to which every element in $\mathfrak{S}_{\mathbb{D}}$ transforms under $\pi^{*}\left(K^{*}\right)$. Let $M$ be an upper bound for $\left|\pi^{*}\left(u^{*}\right)\right|$ on the compact set $K^{*}$. Then it is clear that

$$
\left|E_{\mathfrak{D}}\right| \leqq d(\mathfrak{D})^{2} M \text {. }
$$

Let $q$ and $s$ be two integers $\geqq 0$. Then

$$
\left|E_{\mathfrak{D}} \pi\left(z_{0}^{q+1} w^{\prime}\right) \psi\right| \leqq M d(\mathfrak{D})^{2}\left|\pi\left(z_{0}^{q+1} w^{0}\right) \psi\right| .
$$

But if $X \in \mathfrak{l}_{0}$,

$$
\operatorname{Lim}_{t \rightarrow 0} \frac{1}{t}(\pi(\exp t X)-1) E_{\mathscr{D}} \psi=\operatorname{Lim}_{t \rightarrow 0} E_{\mathbb{D}} \frac{1}{t}(\pi(\exp t X)-1) \psi=E_{\mathscr{D}} \pi(X) \psi
$$

since $E_{\triangleright}$ commutes with $\pi(u)(u \in K)$. Hence it follows that $E_{\triangleright} \psi$ is differentiable under $\pi(K)$ and $\pi(x) E_{\triangleright} \psi=E_{\triangleright} \pi(x) \psi(x \in \mathfrak{X})$. Therefore

$$
E_{D} \pi\left(z_{0}^{q+1} w^{\natural}\right) \psi=\pi\left(z_{0}^{q+1} w^{\natural}\right) E_{D} \psi=d(\mathfrak{D})^{2 q+2}\left|n_{D}\right|^{2 s} E_{D} \psi
$$

since $E_{\mathscr{D} \psi} \psi$ transforms under $\pi(K)$ according to $\mathfrak{D}$. Hence

$$
d(\mathfrak{D})^{2 q}\left|n_{D}\right|^{2 \varepsilon}\left|E_{D} \psi\right| \leqq M\left|\pi\left(z_{0}^{q+1} w^{\delta}\right) \psi\right| \quad\left(\mathfrak{D} \in \Omega_{\pi}\right),
$$

and therefore

$$
\sum_{D \in \Omega_{\pi}}\left|E_{D} \psi\right| \leqq\left(\sum_{D \in \Omega_{\pi}} d(\mathfrak{D})^{-2 q}\left|n_{D}\right|^{-28}\right) M\left|\pi\left(z_{0}^{q+1} w^{\circ}\right) \psi\right| .
$$

For any $\mathfrak{D} \in \Omega_{\pi}$ let $\mathfrak{D}^{\prime}$ denote the class of representations of $\mathfrak{l}^{\prime}$ defined as follows. If $\sigma \in \mathfrak{D}, \mathfrak{D}^{\prime}$ is the class of the restriction of $\sigma$ on $\mathfrak{l}^{\prime}$. Clearly $\mathfrak{D}^{\prime}$ is irreducible and $d\left(\mathfrak{D}^{\prime}\right)=d(\mathfrak{D})$. Moreover $\mathfrak{D}$ is completely determined by $\mathfrak{D}^{\prime}$ and $n_{\mathfrak{D}}$. Hence

$$
\sum_{D \in \Omega_{\pi}} d(\mathfrak{D})^{-2 q}\left|n_{\mathcal{D}}\right|^{-2 s} \leqq \sum_{\mathcal{D}^{\prime}} d\left(\mathfrak{D}^{\prime}\right)^{-2 q} \sum_{n_{1}, \cdots, n_{r}}\left(1+n_{1}^{2}+\cdots+n_{r}^{2}\right)^{-s}
$$

where $\mathfrak{D}^{\prime}$ runs over all irreducible classes of finite-dimensional representations of $\mathfrak{l}^{\prime}$. But if $2 q$ exceeds the rank of $\mathfrak{l}^{\prime}$ it follows from Lemma 2 that $\sum_{\mathfrak{D}}^{\prime} d\left(\mathfrak{D}^{\prime}\right)^{-2 q}<\infty$. Similarly if $2 s>r$

$$
\sum_{(n)}\left(1+n_{1}^{2}+\cdots+n_{r}^{2}\right)^{-s} \leqq \sum_{(n)}\left(1+n_{1}^{2}+\cdots+n_{r}^{2}\right)^{-(r+1) / 2}<\infty .
$$

(9) Conj (x) means conjugate of $x$. 
Therefore if we choose $q$ and $s$ sufficiently large and put

$$
z=N z_{0}^{q+1} w^{8}
$$

where

$$
\begin{gathered}
N=M \sum_{\mathscr{D} \in \Omega_{\pi}} d(\mathfrak{D})^{-2 q}\left|n_{\mathfrak{D}}\right|^{-2 s}, \\
\sum_{\mathscr{D} \in \Omega}\left|E_{\mathscr{D}} \psi\right| \leqq|\pi(z) \psi| .
\end{gathered}
$$

This proves the first assertion of the lemma. Now we come to the second part. Since $\sum_{\mathscr{D} \in \Omega}\left|E_{D} \psi\right|<\infty$ the series $\sum_{\mathscr{D} \in \Omega} E_{D} \psi$ is convergent. Let $\phi$ denote its sum. We have to show that $\phi=\psi$. Put $\psi^{\prime}=\psi-\phi$. Since $E_{D} \phi=E_{D} \psi, E_{D} \psi^{\prime}$ $=0$. From this we shall deduce that $\psi^{\prime}=0$.

Suppose $\psi^{\prime} \neq 0$. Then given any real $\epsilon>0$ choose a continuous real nonnegative function $f$ on $K^{*}$ such that $f\left(u^{*}\right)=0$ if $\left|\pi^{*}\left(u^{*}\right) \psi^{\prime}-\psi^{\prime}\right|>\epsilon\left|\psi^{\prime}\right|$ $\left(u^{*} \in K^{*}\right)$ and $\int_{K^{*}} f\left(u^{*}\right) d u^{*}=1$. Moreover choose a finite linear combination $\omega$ of the matrix coefficients of finite-dimensional simple representations of $K^{*}$ such that $\left|f\left(u^{*}\right)-\omega\left(u^{*}\right)\right| \leqq \epsilon\left(u^{*} \in K^{*}\right)$. Then if

$$
\psi^{\prime \prime}=\int \omega\left(u^{*}\right) \pi^{*}\left(u^{*}\right) \psi^{\prime} d u^{*},
$$

$\psi^{\prime \prime} \in \sum_{\mathfrak{D} \in \Omega} \mathfrak{S}_{\mathfrak{D}}$ and therefore $\psi^{\prime \prime}=\sum_{\mathfrak{D} \in \Omega} E_{\mathfrak{D}} \psi^{\prime \prime}$. But

$$
E_{D} \psi^{\prime \prime}=\int \omega\left(u^{*}\right) \pi^{*}\left(u^{*}\right) E_{D} \psi^{\prime} d u^{*}=0
$$

since $E_{\mathfrak{D}} \psi^{\prime}=0$. Hence $\psi^{\prime \prime}=0$. On the other hand

$$
\begin{aligned}
\left|\psi^{\prime \prime}-\psi^{\prime}\right| \leqq & \int\left|\omega\left(u^{*}\right)-f\left(u^{*}\right)\right|\left|\pi^{*}\left(u^{*}\right) \psi^{\prime}\right| d u^{*} \\
& +\int f\left(u^{*}\right)\left|\pi^{*}\left(u^{*}\right) \psi^{\prime}-\psi^{\prime}\right| d u^{*} \\
\leqq & \leqq \\
&
\end{aligned}
$$

where $M=\sup _{u^{*} \in K^{*}}\left|\pi^{*}\left(u^{*}\right)\right|$. Therefore if $\epsilon$ is sufficiently small

$$
\left|\psi^{\prime}\right|=\left|\psi^{\prime \prime}-\psi^{\prime}\right| \leqq\left|\psi^{\prime}\right| / 2
$$

which contradicts our assumption that $\psi^{\prime} \neq 0$. Therefore $\psi^{\prime}=0$ and so $\sum_{D \in \Omega} E_{D} \psi$ converges to $\psi$.

5. Characters. Let $C_{c}^{\infty}(G)$ denote the class of all complex-valued functions on $G$ which are indefinitely differentiable everywhere and which vanish outside a compact set. Let $\pi$ be a quasi-simple irreducible representation of $G$ on a Hilbert space $\mathfrak{S}$. For any $f \in C_{c}^{\infty}(G)$ consider the operator 


$$
T_{f}=\int f(x) \pi(x) d x
$$

where $d x$ is the Haar measure on $G$. We intend to show that $T_{f}$ is of the trace class.

Let $\mathfrak{N}^{\prime}$ be the Banach space of all bounded linear operators $A$ on $\mathfrak{S}$ with the usual norm $|A|=\sup _{|\psi| \leqq 1}|A \psi|(\psi \in \mathfrak{S})$. Let $\mathfrak{N}_{0}$ be the subspace of $\mathfrak{O}^{\prime}$ consisting of all operators of the form $T_{f}\left(f \in C_{c}^{\infty}(G)\right)$. We denote by $\mathfrak{D}$ the closure of $\mathfrak{D}_{0}$ in $\mathfrak{D}^{\prime}$. Now if $y \in G$,

$$
\pi(y) T_{f}=\int f\left(y^{-1} x\right) \pi(x) d x, \quad T_{f} \pi\left(y^{-1}\right)=\int f(x y) \pi(x) d x .
$$

Hence it follows that if $A \in \mathfrak{D}$ then $\pi(y) A$ and $A \pi\left(y^{-1}\right)$ are also in $\mathfrak{D}$. We now define two representations $l$ and $r$ of $G$ on $\mathcal{D}$ as follows:

$$
l(x) A=\pi(x) A, \quad r(x) A=A \pi\left(x^{-1}\right) \quad(x \in G, A \in \mathfrak{O}) .
$$

In order to verify the conditions for continuity it is sufficient to prove that $\lim _{x \rightarrow 1, y \rightarrow 1}\left|\pi(x) A \pi\left(y^{-1}\right)-A\right|=0 \quad(A \in \mathfrak{D})$. This is done as follows. Given $\epsilon>0$, choose $f \in C_{c}^{\infty}(G)$ such that $\left|A-T_{f}\right| \leqq \epsilon$. Let $U=U^{-1}$ be a compact neighbourhood of 1 in $G$ and $M$ an upper bound for $|\pi(z)|$ for $z \in U$. Then

$$
\left|\pi(x) A \pi\left(y^{-1}\right)-\pi(x) T_{f} \pi\left(y^{-1}\right)\right| \leqq M^{2} \epsilon \quad(x, y \in U)
$$

and therefore

$$
\begin{aligned}
\left|\pi(x) A \pi\left(y^{-1}\right)-A\right| & \leqq\left(M^{2}+1\right) \epsilon+\left|\pi(x) T_{f} \pi\left(y^{-1}\right)-T_{f}\right| \\
& \leqq\left(M^{2}+1\right) \epsilon+\int\left|f\left(x^{-1} z y\right)-f(z)\right||\pi(z)| d z .
\end{aligned}
$$

Let $C$ be a compact set outside which $f$ is zero. We can choose a neighbourhood $V$ of 1 in $G(V \subset U)$ such that $\left|f\left(x^{-1} z y\right)-f(z)\right| \leqq \epsilon$ if $x, y \in V$. Let $F$ be a real nonnegative continuous function on $G$ which is equal to 1 on $C$ and which vanishes outside some compact set. Then if $N_{0}=\sup _{z \in U C U}|\pi(z)|$,

$$
\left|\pi(x) A \pi\left(y^{-1}\right)-A\right| \leqq\left(M^{2}+1\right) \epsilon+N_{0} \epsilon \int F(z) d z
$$

provided $x, y \in V$. This proves that $\lim _{x \rightarrow 1, y \rightarrow 1}\left|\pi(x) A \pi\left(y^{-1}\right)-A\right|=0$.

Since $l(x) T_{f}=\int f\left(x^{-1} z\right) \pi(z) d z$, it follows easily that $T_{f}$ is differentiable under $l$. Similarly we show that it is differentiable under $r$. It is clear that the representations $l$ and $r$ are permissible. For any $\mathfrak{D} \in \Omega$ let $E_{\mathfrak{D}}, P_{\mathfrak{D}}$, and $Q_{\mathfrak{D}}$ denote the canonical projections (see $\S 9$ of [6]) corresponding to $\mathfrak{D}$ under $\pi, l$, and $r$ respectively. Then it is clear $P_{D} A=E_{D} A ; Q_{D} A=A E_{D^{\prime}}(\mathfrak{D} \in \Omega)$ where $\mathfrak{D}^{\prime}$ is the class contragredient to $\mathfrak{D}$. Let $\lambda$ and $\rho$ denote the left and right regular representations of $G$. Then every element in $C_{c}^{\infty}(G)$ is differentiable under both $\lambda$ and $\rho$ and $C_{c}^{\infty}(G)$ is invariant under $\lambda(\mathfrak{B})$ and $\rho(\mathfrak{B})$. Moreover 
since $l(x) r(y) T_{f}=T_{\lambda(x) \rho(y) f}(x, y \in G)$ it follows easily that $l(a) r(b) T_{f}$ $=T_{\lambda(a) \rho(b) f}(a, b \in \mathfrak{B})$.

Now define a representation $\phi$ of the group $G \times G$ on $\supseteqq$ as follows. $\phi(x, y) A$ $=l(x) r(y) A=\pi(x) A \pi\left(y^{-1}\right)(x, y \in G)$. Then $\phi$ is a permissible representation of the semisimple group $G \times G$ and any element of $\mathfrak{D}_{0}$ is differentiable under $\phi$. Moreover the canonical projections for the representation $\phi$ (with respect to the subgroup $K \times K)$ are exactly the operators $P_{\mathfrak{D}_{1}} Q_{\mathfrak{D}_{2}}\left(\mathfrak{D}_{1}, \mathfrak{D}_{2} \in \Omega\right)$. Let $z_{0}$ be the element of $\mathfrak{X}$ which was introduced in the proof of Lemma 3. Then if we apply Lemma 3 to the representation $\phi$ and the differentiable element $T_{\lambda\left(z_{0}\right) \rho\left(z_{0}\right) f}$ we find that

$$
\sum_{D_{1}, \mathscr{D}_{2} \in \Omega}\left|P_{\mathscr{D}_{1}} Q_{\mathfrak{D}_{2}} T_{\lambda\left(z_{0}\right) \rho\left(z_{0}\right) f}\right|<\infty .
$$

But

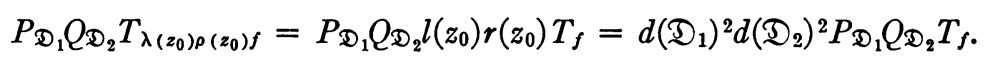

Hence

$$
\sum_{\mathfrak{D}_{1}, \mathfrak{D}_{2} \in \Omega} d\left(\mathfrak{D}_{1}\right)^{2} d\left(\mathfrak{D}_{2}\right)^{2}\left|E_{\mathfrak{D}_{1}} T_{f} E_{\mathfrak{D}_{2}}\right|<\infty .
$$

Now let us first suppose that the subspaces $\mathfrak{S}_{\mathfrak{D}}=E_{\mathfrak{D}} \mathfrak{S}(\mathfrak{D} \in \Omega)$ are mutually orthogonal. Choose an orthonormal base for each $\mathfrak{S C D}_{\mathfrak{D}}$. All these put together form an orthonormal base $\left\{\psi_{\alpha}\right\}_{\alpha \in J}$ for $\mathfrak{S}$. In accordance with Theorem 4 we choose an integer $N$ such that $\operatorname{dim} \mathfrak{S}_{\mathfrak{D}} \leqq N d(\mathfrak{D})^{2}(\mathfrak{D} \in \Omega)$. Then

$$
\sum_{\alpha, \beta \in J}\left|\left(\psi_{\alpha}, T_{f} \psi_{\beta}\right)\right|=\sum_{D_{1}, D_{2} \in \Omega} \sum_{\alpha \in J_{D_{1}}} \sum_{\beta \in J_{D_{2}}}\left|\left(\psi_{\alpha}, T_{f} \psi_{\beta}\right)\right|
$$

where $J_{\mathscr{D}}$ is the subset of $J$ such that $\left\{\psi_{\alpha}\right\}_{\alpha \in J_{\mathfrak{D}}}$ is a base for $\mathfrak{F}_{\mathfrak{D} D}$. But it is clear that

$$
\begin{aligned}
\sum_{\alpha \in J_{\mathfrak{D}_{1}}} \sum_{\beta \in J_{D_{2}}}\left|\left(\psi_{\alpha}, T_{f} \psi_{\beta}\right)\right| & \leqq \operatorname{dim} \mathfrak{W}_{\mathfrak{D}_{1}} \operatorname{dim} \mathfrak{S}_{\mathscr{D}_{2}}\left|E_{\mathfrak{D}_{1}} T_{f} E_{\mathfrak{D}_{2}}\right| \\
& \leqq N^{2} d\left(\mathfrak{D}_{1}\right)^{2} d\left(\mathfrak{D}_{2}\right)^{2}\left|E_{\mathfrak{D}_{1}} T_{f} E_{\mathfrak{D}_{2}}\right| .
\end{aligned}
$$

Hence

$$
\sum_{\alpha, \beta \in J}\left|\psi_{\alpha}, T_{f} \psi_{\beta}\right| \leqq N^{2} \sum_{\mathfrak{D}_{1}, \mathscr{D}_{2} \in \Omega} d\left(\mathfrak{D}_{1}\right)^{2} d\left(\mathfrak{D}_{2}\right)^{2}\left|E_{\mathfrak{D}_{1}} T_{f} E_{\mathfrak{D}_{2}}\right|<\infty
$$

and therefore, from Lemma $1, T_{f}$ is of the trace class.

Now we discard the assumption about the mutual orthogonality of the spaces $\mathfrak{S C D}_{\mathfrak{D}}$. Let $x \rightarrow x^{*}$ denote the natural mapping of $G$ on $G^{*}=G / D \cap Z$ Define a representation $\pi^{*}$ of $K^{*}$ on $\mathfrak{S}$ as in the proof of Lemma 3. Since $K^{*}$ is a compact group, $\pi^{*}$ is equivalent to a unitary representation. Hence there exists a regular operator $B$ on $\mathfrak{S}$ such that the representation $\pi^{*}$ : $u$ $\rightarrow B \pi^{*}\left(u^{*}\right) B^{-1}\left(u^{*} \in K^{*}\right)$ is unitary. Now put $\pi^{\prime}(x)=B \pi(x) B^{-1}$. Then $\pi^{\prime}$ is a 
representation of $G$ on $\mathfrak{S}$. Let $\mathfrak{W}_{\mathfrak{D}}^{\prime}$ be the subspace of $\mathfrak{E}$ consisting of all elements which transform under $\pi^{\prime}(K)$ according to $\mathfrak{D}(\mathscr{D} \in \Omega)$. Then since $\pi^{\prime *}$ is unitary the spaces $\mathfrak{S}_{\mathfrak{D}}^{\prime}$ are mutually orthogonal. Therefore the above proof is applicable to

$$
T_{f}^{\prime}=\int f(x) \pi^{\prime}(x) d x=B T_{f} B^{-1} .
$$

Hence $T_{f}^{\prime}$ fulfills the condition of Lemma 1 and therefore $T_{f}=B^{-1} T_{f} B$ is of the trace class. Moreover if $P$ and $Q$ are two bounded operators on $\mathfrak{S}$, then $P B^{-1}$ and $B Q$ are also bounded and $P T_{f} Q=\left(P B^{-1}\right) T_{f}^{\prime}(B Q)$. Therefore, from Lemma 1, $P T_{f} Q, T_{f} Q P, Q P T_{f}$ are all of the trace class and their traces are equal. Therefore in particular sp $\left(A T_{f} A^{-1}\right)=\operatorname{sp} T_{f}$ if $A$ is a regular operator.

Put $T_{\pi}(f)=\operatorname{sp}\left(T_{f}\right)$ for any $f \in C_{c}^{\infty}(G)$. Then $T_{\pi}$ is a linear function on the vector space $C_{c}^{\infty}(G)$. Furthermore if $a$ is a fixed element in $G$ and $g$ is the function $g(x)=f\left(a x a^{-1}\right)(x \in G)$ then

$$
T_{g}=\pi\left(a^{-1}\right) T_{f} \pi(a)
$$

and therefore $T_{\pi}(g)=T_{\pi}(f)$. Hence we may say that $T_{\pi}$ is invariant under the inner automorphisms of $G$. We prove similarly that if $\pi$ and $\pi^{\prime}$ are equivalent representations, $T_{\pi}=T_{\pi^{\prime}}$.

Our next object is to show that $T_{\pi}$ is actually a distribution in the sense of L. Schwartz [9]. By going over to an equivalent representation, if necessary, we may assume that the spaces $\mathfrak{S}_{\mathfrak{D}}(\mathfrak{D} \in \Omega)$ are mutually orthogonal. Then it is clear that

$$
\operatorname{sp} T_{f}=\sum_{\mathfrak{D} \in \Omega} \operatorname{sp}\left(E_{\mathfrak{D}} T_{f} E_{\mathfrak{D}}\right)
$$

and

$$
\operatorname{sp}\left(E_{\mathfrak{D}} T_{f} E_{\mathfrak{D}}\right)=\sum_{i=1}^{d}\left(\psi_{i}, E_{\mathfrak{D}} T_{f} \psi_{i}\right)
$$

where $\left(\psi_{1}, \cdots, \psi_{d}\right)$ is an orthonormal base of $\mathfrak{S E D}_{\mathfrak{D}}$. Therefore

$$
\left|\operatorname{sp}\left(E_{\mathfrak{D}} T_{f} E_{\mathfrak{D}}\right)\right| \leqq \operatorname{dim} \mathfrak{S}_{\mathfrak{D}}\left|E_{\mathfrak{D}} T_{f}\right| \leqq N d(\mathfrak{D})^{2}\left|E_{\mathfrak{D}} T_{f}\right|
$$

and

$$
\left|T_{\pi}(f)\right| \leqq N \sum_{\mathcal{D} \in \Omega} d(\mathfrak{D})^{2}\left|E_{\mathscr{D}} T_{f}\right|=N \sum_{\mathcal{D} \in \Omega}\left|E_{\mathfrak{D}} T_{\lambda\left(z_{0}\right) f}\right|
$$

where $z_{0}$ has the same meaning as above. Now by applying Lemma 3 to the representation $l$ of $G$ on $\mathfrak{D}$ and the differentiable element $T_{\lambda\left(z_{0}\right) f}$ we conclude that

$$
\sum_{D \in \Omega}\left|E_{\overparen{D}} T_{\lambda\left(z_{0}\right) f}\right| \leqq\left|l(z) T_{\lambda\left(z_{0}\right) f}\right|=\left|T_{\lambda\left(z z_{0}\right) f}\right|
$$


where $z$ is an element of $\mathfrak{X}$ (which does not depend on $f$ ). Hence

$$
\left|T_{\pi}(f)\right| \leqq N\left|T_{\lambda\left(z z_{0}\right) f}\right| \text {. }
$$

Now suppose $C$ is a compact set in $G$ and $f_{n}$ is a sequence of functions in $C_{c}^{\infty}(G)$ such that $f_{n}$ vanishes outside $C$ and for any $b \in \mathfrak{B}, \lambda(b) f_{n} \rightarrow 0$ uniformly on $C$. Then

$$
\left|T_{\lambda(b) f_{n}}\right| \leqq \int\left|\left(\lambda(b) f_{n}\right)(x)\right||\pi(x)| d x \rightarrow 0
$$

and therefore in particular $\left|T_{\pi}\left(f_{n}\right)\right| \leqq N\left|T_{\lambda\left(z z_{0}\right) f_{n}}\right| \rightarrow 0$. This proves that $T_{\pi}$ is a distribution.

6. Operators of the Hilbert-Schmidt class. Let $B$ be a bounded operator on the Hilbert space $\mathfrak{S}$ and let $B^{*}$ be the adjoint of $B$. We say that $B$ is of the Hilbert-Schmidt (H.S.) class if $B^{*} B$ has a trace. Let $\left\{\psi_{\alpha}\right\}_{\alpha \in J}$ be an orthonormal base for $\mathfrak{S}$. Then it is well known that $\|B\|^{2}=\sum_{\alpha \in J}\left|B \psi_{\alpha}\right|^{2}$ is independent of the choice of this base and $B$ is of the H.S. class if and only if $\|B\|<\infty$. Moreover sp $B B^{*}=\|B\|^{2}=\left\|B^{*}\right\|^{2}$ if $\|B\|<\infty$ and $\left\|A_{1} B A_{2}\right\|$ $\leqq\left|A_{1}\right|\|B\|\left|A_{2}\right|$ for any two bounded operators $A_{1}, A_{2}$.

Let $\pi$ be a quasi-simple irreducible representation of $G$ on $\mathfrak{S}$. Let $f$ be a complex-valued measurable function on $G$ which vanishes outside a compact set and such that $\int|f(x)|^{2} d x<\infty$. It follows from the Schwartz inequality that $\int|f(x)| d x<\infty$ and therefore the operator $\int f(x) \pi(x) d x$ is a well-defined bounded operator. We intend to prove that this operator is of the H.S. class. Let $S$ be a regular operator on $\mathfrak{S}$. Put $\pi^{\prime}(x)=S \pi(x) S^{-1}(x \in G)$. Then

$$
\left\|\int f(x) \pi(x) d x\right\|=\left\|S^{-1} \int f(x) \pi^{\prime}(x) d x S\right\| \leqq\left|S^{-1}\right|\left\|\int f(x) \pi(x) d x\right\||S| .
$$

Therefore it is enough to show that the corresponding operator for an equivalent representation is of the H.S. class.

Let $x \rightarrow x^{*}$ denote the natural mapping of $G$ on $G^{*}=G / D \cap Z$. For any $x \in G$ we define $\Gamma(x)$ to be the unique element in $\mathfrak{c}_{0}$ such that $x=u(\exp \Gamma(x)) s$ where $u \in K^{\prime}$ and $s$ lies in the solvable subgroup of $G$ corresponding to the subalgebra $\mathfrak{g}_{0} \cap\left(\mathfrak{h}_{\mathfrak{p}}+\mathfrak{n}\right)$ of $\mathfrak{g}_{0}$ (see $\left.[6, \S 9]\right)$. Then if $\mu$ is the linear function on $\mathfrak{c}$ which was introduced in the proof of Lemma 3 , it is clear that $\pi(x) e^{-\mu(\Gamma(x))}$ depends only on $x^{*}$. Put $\pi^{*}\left(x^{*}\right)=\pi(x) e^{-\mu(\Gamma(x))}$. Then we verify immediately that $\pi^{*}\left(u^{*} x^{*}\right)=\pi^{*}\left(u^{*}\right) \pi^{*}\left(x^{*}\right)\left(u^{*} \in K^{*}, x^{*} \in G^{*}\right)$ and therefore $u^{*} \rightarrow \pi^{*}\left(u^{*}\right)$ is a representation of $K^{*}$. In view of the preceding remarks we may assume without loss of generality that this representation is unitary.

Let $x^{*} \in G^{*}$ and $y \in G$. We say that $y$ lies above $x^{*}$ and write $y>x^{*}$ if $(y)^{*}=x^{*}$. Put

$$
f^{*}\left(x^{*}\right)=\sum_{x>x^{*}} e^{\mu(\Gamma(x))} f(x)
$$$$
\left(x^{*} \in G^{*}\right) .
$$ 
Let $A$ be a compact set outside which $f$ is zero. Since $D \cap Z$ is discrete, $(D \cap Z) \cap A^{-1} A$ is a finite set. Let $N_{0}$ be the number of elements in it. Then it is clear that not more than $N_{0}$ distinct elements in $A$ can lie above the same element in $G^{*}$. Hence at most $N_{0}$ terms in the above sum are different from zero and therefore the function $f^{*}$ is well-defined. Moreover if $A^{*}$ is the image of $A$ in $G^{*}, f^{*}$ is zero outside $A^{*}$. Now let $x^{*} \in A^{*}$. Then

$$
\begin{aligned}
\left|f^{*}\left(x^{*}\right)\right| & =\left|\sum_{x>x^{*}} e^{\mu(\Gamma(x))} f(x)\right| \leqq\left(\sum_{x>x^{*}}|f(x)|^{2}\right)^{1 / 2}\left(\sum_{y>x^{*}}\left|e^{\mu(\Gamma(y))}\right|^{2}\right)^{1 / 2} \\
& \leqq M_{0} N_{0}^{1 / 2}\left(\sum_{x>x^{*}}|f(x)|^{2}\right)^{1 / 2}
\end{aligned}
$$

where $M_{0}=\sup _{y \in A}\left|e^{\mu(\Gamma(y))}\right|$. Hence if the Haar measure $d x^{*}$ on $G^{*}$ is suitably normalised it follows that

$$
\int f(x) \pi(x) d x=\int f^{*}\left(x^{*}\right) \pi^{*}\left(x^{*}\right) d x^{*}
$$

and

$$
\int\left|f^{*}\left(x^{*}\right)\right|^{2} d x^{*} \leqq M_{0}^{2} N \int|f(x)|^{2} d x<\infty .
$$

Let $B^{*}$ be a compact neighbourhood of $A^{*}$. Choose a real-valued nonnegative function $F$ on $G^{*}$ such that $F=1$ on $K^{*} B^{*}$ and $F=0$ outside some compact set. Let $g$ be any continuous function on $G^{*}$ which vanishes outside $B^{*}$. Consider the operator

$$
\int g\left(x^{*}\right) \pi\left(x^{*}\right) d x^{*}=\int d u^{*} \int g\left(u^{*} x^{*}\right) \pi^{*}\left(u^{*} x^{*}\right) d x^{*} .
$$

(Here $d u^{*}$ is the normalised Haar measure on $K^{*}$ so that $\int d u^{*}=1$.) Then

$$
\left\|\int g\left(x^{*}\right) \pi^{*}\left(x^{*}\right) d x^{*}\right\| \leqq \int d x^{*}\left\|\int g\left(u^{*} x^{*}\right) \pi^{*}\left(u^{*} x^{*}\right) d u^{*}\right\| .
$$

But

$$
\left\|\int g\left(u^{*} x^{*}\right) \pi^{*}\left(u^{*} x^{*}\right) d u^{*}\right\| \leqq\left\|\int g\left(u^{*} x^{*}\right) \pi^{*}\left(u^{*}\right) d u^{*}\right\|\left|\pi^{*}(x)\right|
$$

and from Theorem 4 we can find an integer $N$ such that $\operatorname{dim} \mathfrak{S}_{\mathfrak{D}} \leqq N d(\mathfrak{D})^{2}$ for any $\mathfrak{D} \in \Omega$. Let $\Omega^{*}$ be the set of all classes of irreducible finite-dimensional representations of $K^{*}$. Then no $\mathfrak{D}^{*} \in \Omega^{*}$ occurs more than $N d\left(\mathfrak{D}^{*}\right)$ times in the reduction of $\pi^{*}\left(K^{*}\right)$. Since every $\mathfrak{D}^{*} \in \Omega^{*}$ occurs exactly $d\left(\mathfrak{D}^{*}\right)$ times in the left regular representation $\lambda$ of $K^{*}$ (on the Hilbert space $L_{2}\left(K^{*}\right)$ of all 
square integrable functions on $\left.K^{*}\right)$ and since the representation $u^{*} \rightarrow \pi^{*}\left(u^{*}\right)$ $\left(u^{*} \in K^{*}\right)$ is unitary, we may conclude that

$$
\left\|\int g\left(u^{*} x^{*}\right) \pi^{*}\left(u^{*}\right) d u^{*}\right\|^{2} \leqq N\left\|\int g\left(u^{*} x^{*}\right) \lambda\left(u^{*}\right) d u^{*}\right\|^{2} .
$$

But from the Peter-Weyl theorem we know that

$$
\left\|\int g\left(u^{*} x^{*}\right) \lambda\left(u^{*}\right) d u^{*}\right\|^{2}=\int\left|g\left(u^{*} x^{*}\right)\right|^{2} d u^{*} .
$$

Hence

$$
\left\|\int g\left(u^{*} x^{*}\right) \pi^{*}\left(u^{*}\right) d u^{*}\right\| \leqq N^{1 / 2}\left(\int\left|g\left(u^{*} x^{*}\right)\right|^{2} d u^{*}\right)^{1 / 2} .
$$

Now it is easy to see that $\left|\pi^{*}\left(x^{*}\right)\right|$ is bounded on the compact set $K^{*} B^{*}$. Let $M=\sup _{x^{*} \in K^{*} B^{*}}\left|\pi^{*}\left(x^{*}\right)\right|$. Then

$$
\left\|\int g\left(u^{*} x^{*}\right) \pi^{*}\left(u^{*} x^{*}\right) d u^{*}\right\| \leqq M N^{1 / 2}\left(\int\left|g\left(u^{*} x^{*}\right)\right|^{2} d u^{*}\right)^{1 / 2}
$$

and therefore

$$
\begin{aligned}
\left\|\int g\left(x^{*}\right) \pi^{*}\left(x^{*}\right) d x^{*}\right\| & \leqq M N^{1 / 2} \int d x^{*}\left(\int\left|g\left(u^{*} x^{*}\right)\right|^{2} d u^{*}\right)^{1 / 2} \\
& =M N^{1 / 2} \int F\left(x^{*}\right) d x^{*}\left(\int\left|g\left(u^{*} x^{*}\right)\right|^{2} d u^{*}\right)^{1 / 2} \\
& \leqq M_{1}\left(\int\left|g\left(x^{*}\right)\right|^{2} d x^{*}\right)^{1 / 2}
\end{aligned}
$$

where $M_{1}=M N^{1 / 2}\left(\int\left|F\left(x^{*}\right)\right|^{2} d x^{*}\right)^{1 / 2}$. Now choose a sequence $g_{n}$ of continuous functions on $G^{*}$ which vanish outside $B^{*}$ and such that $\int\left|f^{*}\left(x^{*}\right)-g_{n}\left(x^{*}\right)\right|^{2} d x^{*}$ $\rightarrow 0$. Then since

$$
\begin{aligned}
\int\left|f^{*}\left(x^{*}\right)-g_{n}\left(x^{*}\right)\right| & d x^{*} \\
& \leqq\left(\int\left|F\left(x^{*}\right)\right|^{2} d x^{*}\right)^{1 / 2}\left(\int\left|f^{*}\left(x^{*}\right)-g_{n}\left(x^{*}\right)\right|^{2} d x^{*}\right)^{1 / 2}
\end{aligned}
$$

it follows that

$$
\left|\int\left(f^{*}\left(x^{*}\right)-g_{n}\left(x^{*}\right)\right) \pi^{*}\left(x^{*}\right) d x^{*}\right| \rightarrow 0 .
$$

Moreover we have seen above that 


$$
\left\|\int\left(g_{m}\left(x^{*}\right)-g_{n}\left(x^{*}\right)\right) \pi^{*}\left(x^{*}\right) d x^{*}\right\| \leqq M_{1}\left(\int\left|g_{m}\left(x^{*}\right)-g_{n}\left(x^{*}\right)\right|^{2} d x^{*}\right)^{1 / 2}
$$

and therefore the sequence of operators $T_{n}=\int g_{n}\left(x^{*}\right) \pi^{*}\left(x^{*}\right) d x^{*}$ is a Cauchy sequence with respect to the Hilbert-Schmidt norm \|\| . Since the space of operators of the H.S. class is complete with respect to this norm, there exists an operator $T$ of this class such that $\left\|T_{n}-T\right\| \rightarrow 0$. But $\left|T_{n}-T\right|$ $\leqq\left\|T_{n}-T\right\|$. Hence $\left|T_{n}-T\right| \rightarrow 0$. However we have seen already that

$$
\left|T_{n}-\int f^{*}\left(x^{*}\right) \pi^{*}\left(x^{*}\right) d x^{*}\right| \rightarrow 0
$$

and therefore $T=\int f^{*}\left(x^{*}\right) \pi^{*}\left(x^{*}\right) d x^{*}$. This proves that $T=\int f(x) \pi(x) d x$ is of the H.S. class. Thus we have the following theorem $\left({ }^{10}\right)$.

THEOREM 5. Let $\pi$ be a quasi-simple irreducible representation of $G$ on a Hilbert space $\mathfrak{S}$ and let $f$ be a measurable and square integrable function on $G$ which vanishes outside a compact set. Then the operator $\int f(x) \pi(x) d x$ is of the Hilbert-Schmidt class.

7. Linear independence of characters. Let $T_{\pi}$ be the character of a quasisimple irreducible representation $\pi$ of $G$ on a Hilbert space $\mathfrak{S}$. Let $E_{\mathscr{D}}$ denote the canonical projection of $\mathfrak{S}$ on $\mathfrak{S E D}_{\mathfrak{D}}(\mathfrak{D} \in \Omega)$. Then it follows easily from its definition (see \$5) that

$$
\begin{aligned}
T_{\pi}(f)=\operatorname{sp}\left(\int f(x) \pi(x) d x\right) & =\sum_{\mathscr{D} \in \Omega} \operatorname{sp}\left(E_{\mathfrak{D}} \int f(x) \pi(x) d x \cdot E_{\mathfrak{D}}\right) \\
& =\sum_{\mathfrak{D} \in \Omega} \int f(x) \phi_{\mathfrak{D}}^{\pi}(x) d x
\end{aligned}
$$

in the notation of $\S 1$. Now if $\pi_{1}, \pi_{2}$ are two infinitesimally equivalent representations, we have seen in $\S 1$ that $\phi_{\mathfrak{D}}^{\pi_{1}}=\phi_{\mathfrak{D}}^{\pi_{2}}(\mathfrak{D} \in \Omega)$ and therefore $T_{\pi_{1}}=T_{\pi_{2}}$. Hence two infinitesimally equivalent quasi-simple irreducible representations (on Hilbert spaces) have the same character. Conversely we shall show that two such representations having the same character are infinitesimally equivalent( $\left.{ }^{11}\right)$.

THEOREM 6. Let $\pi_{1}, \cdots, \pi_{q}$ be a finite set of quasi-simple irreducible representations of $G$ on the Hilbert spaces $\mathfrak{S}_{1}, \cdots, \mathfrak{L}_{q}$ respectively. Suppose no two of them are infinitesimally equivalent. Then their characters $T_{\pi_{1}}, \cdots, T_{\pi_{q}}$ are linearly independent.

For otherwise suppose $c_{1} T_{\pi_{1}}+\cdots+c_{q} T_{\pi_{q}}=0\left(c_{i} \in C\right)$ where, say, $c_{1} \neq 0$.

(10) Cf. Theorem 4 of $[8(c)]$.

(11) Cf. Theorem 3 of [8(c)]. 
Let $\eta_{i}$ be the nomomorphism of $D \cap Z$ into $C$ such that $\pi_{i}(\gamma)=\eta_{i}(\gamma) \pi_{i}(1)$ $(\gamma \in D \cap Z)$. Then if $f \in C_{c}^{\infty}(G)$ and $\gamma \in D \cap Z$, the function $f_{\gamma}: x \rightarrow f\left(\gamma^{-1} x\right)$ $(x \in G)$ is also in $C_{c}^{\infty}(G)$ and it is obvious that $T_{x_{i}}\left(f_{\gamma}\right)=\eta_{i}(\gamma) T_{x_{i}}(f)$. Therefore $\sum_{i=1}^{q} c_{i} T_{\pi_{i}}\left(f_{\gamma}\right)=\sum_{i=1}^{q} c_{i} \eta_{i}(\gamma) T_{\pi_{i}}(f)=0$. This proves that

$$
\sum_{i=1}^{q} c_{i} \eta_{i}(\gamma) T_{\pi_{i}}=0
$$

for all $\gamma \in D \cap Z$. Now if we recall that $D \cap Z$ is a free abelian group with $r$ generators $\left(r=\operatorname{dim}_{R} \mathfrak{c}_{0}\right)$ we can conclude that $c_{1} T_{\pi_{1}}+\cdots+c_{s} T_{\pi_{s}}=0$ assuming that $\eta_{j}=\eta_{1}(1 \leqq j \leqq s)$ and $\eta_{j} \neq \eta_{i}$ for $s<j \leqq q$.

Choose a base $\Gamma_{1}, \cdots, \Gamma_{r}$ for $\mathfrak{c}_{0}$ over $R$ such that $\exp \Gamma_{i}, 1 \leqq i \leqq r$, is a set of generators for $D \cap Z$. Select a linear function $\mu$ on $c$ such that $\eta_{1}\left(\exp \Gamma_{i}\right)$ $=e^{\mu\left(\Gamma_{i}\right)}, 1 \leqq i \leqq r$. Put $\pi_{j}^{*}\left(x^{*}\right)=e^{-\mu(\Gamma(x))} \pi_{j}(x)(x \in G, 1 \leqq j \leqq s)$ in the notation of $\S 6$. Let $\mathfrak{D}$ be a class in $\Omega$ which occurs in $\pi_{1}$. We denote by $E_{\mathfrak{D}}^{t}$ the canonical projection of $\mathfrak{S}_{i}$ on $\mathfrak{S}_{i}, \mathfrak{D}$. Then

$$
E_{\mathfrak{D}}^{i}=d(\mathfrak{D}) \int \operatorname{conj}\left(\xi_{D^{*}}\left(u^{*}\right)\right) \pi_{i}{ }^{*}\left(u^{*}\right) d u^{*}
$$

where $\mathfrak{D}^{*}$ is the irreducible class according to which every element in $\mathfrak{S}_{1, \mathbb{D}}$ transforms under $\pi_{1}^{*}\left(K^{*}\right)$ and $\xi_{\mathbb{D}^{*}}$ is the character of $\mathfrak{D}^{*}$. Let $K_{0}$ be the set of all elements in $K$ of the form $(\exp \Gamma) v$ where $\Gamma=t_{1} \Gamma_{1}+\cdots+t_{r} \Gamma_{r}\left(t_{j} \in R\right.$, $\left.\left|t_{j}\right| \leqq 1 / 2\right)$ and $v \in K^{\prime}$. Then $K_{0}$ is compact and if we put

$$
\xi_{\mathcal{D}}(u)=e^{-\operatorname{conj}(\mu(\Gamma(u)))} \xi_{\mathbb{D}^{*}}\left(u^{*}\right) \quad(u \in K),
$$

we get

$$
E_{\mathfrak{D}}^{i}=d(\mathfrak{D}) \int_{K_{0}} \operatorname{conj}\left(\xi_{\mathfrak{D}}(u)\right) \pi_{i}(u) d u
$$

where the Haar measure $d u$ on $K$ is so normalised that $\int_{K_{0}} d u=1$.

Now we use the notation of Theorem 1. Put

$$
\phi=c_{1} \phi_{\mathfrak{D}}^{\pi_{1}}+\cdots+c_{8} \phi_{\mathfrak{D}}^{\pi_{1}} .
$$

It follows from Theorem 1 that $\phi \neq 0$. Since $\phi$ is continuous we can find a function $f \in C_{c}^{\infty}(G)$ such that $\int f(x) \phi(x) d x \neq 0$. Now

$$
E_{\mathfrak{D}}^{i} \int f(x) \pi_{i}(x) d x=\int f_{\mathfrak{D}}(x) \pi_{i}(x) d x \quad(1 \leqq i \leqq s)
$$

where

$$
f_{\mathfrak{D}}(x)=d(\mathfrak{D}) \int_{K_{0}} \operatorname{conj}\left(\xi_{\mathfrak{D}}(u)\right) f\left(u^{-1} x\right) d u .
$$

Since $K_{0}$ is compact it is clear that $f_{\triangleright} \in C_{c}^{\infty}(G)$. On the other hand 


$$
\begin{aligned}
\operatorname{sp}\left(\int f_{\mathfrak{D}}(x) \pi_{i}(x) d x\right)= & \operatorname{sp}\left(E_{\mathfrak{D}}^{i} \int f(x) \pi_{i}(x) d x\right) \\
= & \operatorname{sp}\left(E_{\mathfrak{D}}^{i} \int f(x) \pi_{i}(x) d x \cdot E_{\mathfrak{D}}^{i}\right)=\int f(x) \phi_{\mathfrak{D}}^{\pi_{i}}(x) d x .
\end{aligned}
$$

Therefore $c_{1} T_{\pi_{1}}\left(f_{\mathfrak{D}}\right)+\cdots+c_{s} T_{\pi_{s}}\left(f_{\mathfrak{D}}\right)=\int f(x) \phi(x) d x \neq 0$. This however implies that $c_{1} T_{x_{i}}+\cdots+c_{s} T_{x_{s}} \neq 0$ and so we get a contradiction.

COROLlaRy. Two irreducible unitary represeniations are equivalent if and only if their characters are the same.

First of all every irreducible unitary representation is quasi-simple (see for example Segal [10]). Moreover infinitesimal equivalence is the same as ordinary equivalence for two such representations (see Theorem 8 of [6]). Hence the corollary is an immediate consequence of the theorem.

8. Complex semisimple groups. Suppose the group $G$ is complex. Then $K$ is semisimple and there exists a 1-1 linear mapping $i$ of $\mathfrak{t}_{0}$ onto $\mathfrak{p}_{0}$ such that

$$
[X, i(Y)]=i([X, Y]), \quad[i(X), i(Y)]=-[X, Y] \quad\left(X, Y \in \mathfrak{f}_{0}\right) .
$$

Let $\mathfrak{h}_{\mathfrak{t}_{0}}$ be a maximal abelian subalgebra of $\mathfrak{f}_{0}$. Then $i\left(\mathfrak{h}_{\mathfrak{t}_{0}}\right)$ is clearly a maximal abelian subspace of $\mathfrak{p}_{0}$. Hence we may take $\mathfrak{h}_{\mathfrak{p}_{0}}=i\left(\mathfrak{h}_{\mathfrak{p}_{0}}\right)$. Then $\mathfrak{h}_{\mathfrak{f}_{0}}+\mathfrak{h}_{\mathfrak{p}_{0}}$ is a maximal abelian subalgebra of $\mathfrak{g}_{0}$. Let $\mathfrak{h}_{\mathfrak{t}}$ and $\mathfrak{h}_{\mathfrak{p}}$ be the subspaces of $\mathfrak{g}$ spanned by $\mathfrak{h}_{\mathfrak{t}_{0}}$ and $\mathfrak{h}_{\mathfrak{p}_{0}}$ over $C$. Then $\mathfrak{h}=\mathfrak{h}_{\mathfrak{t}}+\mathfrak{h}_{\mathfrak{p}}$ is a Cartan subalgebra of $\mathfrak{g}$. We extend $i$ to a mapping of $\mathfrak{t}$ into $\mathfrak{p}$ by linearity.

Let $\alpha_{1}, \cdots, \alpha_{p}$ be a maximal set of linearly independent roots of $\mathfrak{t}$ (with respect to $\mathfrak{h}_{\mathfrak{t}}$ ). We order all roots $\alpha$ of $\mathfrak{t}$ lexicographically with respect to this set (see [5, Part I]). For every root $\alpha$ let $H_{\alpha}$ be the element in $\mathfrak{h}_{t}$ such that sp $\left(\operatorname{ad}^{\prime} H\right.$ ad $\left.^{\prime} H_{\alpha}\right)=\alpha(H)\left(H \in \mathfrak{h}_{\mathfrak{t}}\right)$ where $X \rightarrow \operatorname{ad}^{\prime} X(X \in \mathfrak{f})$ is the adjoint representation of $\mathfrak{f}$. We denote by $W$ the Weyl group of $\mathfrak{t}$ and by $2 \sigma$ the sum of all positive roots of $\mathfrak{f}$. Let $\lambda$ be a linear function on $\mathfrak{h}_{\mathfrak{r}}$. We put $\lambda^{\prime}=\lambda+\sigma$ and use the notation of [5, Part III]. We know (see [5, p. 70]) that the power series $\sum_{s \in W} \epsilon(s) e^{\lambda^{\prime}(s H)}$ is divisible by $\prod_{\alpha>0} \lambda^{\prime}\left(H_{\alpha}\right) \prod_{\alpha>0} \alpha(H)$ $\left(H \in \mathfrak{h}_{\mathfrak{r}}\right)$ and therefore the quotient

$$
\frac{\prod_{s \in W} \epsilon(s) e^{\lambda^{\prime}(s H)}}{\prod_{\alpha>0} \lambda^{\prime}\left(H_{\alpha}\right) \prod_{\alpha>0} \alpha(H)}
$$

is an analytic function on $\mathfrak{h}_{\mathfrak{r}}$. Similarly

$$
\frac{\prod_{\alpha>0} \alpha(H)}{\prod_{\alpha>0}\left(e^{\alpha(H) / 2}-e^{-\alpha(H) / 2}\right)}
$$


is a meromorphic function on $\mathfrak{h}_{\mathfrak{t}}$ all whose singularities lie on hyperplanes of the form $\alpha(H)=2 \pi(-1)^{1 / 2} n$ where $\alpha$ is a root and $n$ is some nonzero integer. Hence the function

$$
\Phi^{*}(\lambda, H)=\prod_{\alpha>0} \sigma\left(H_{\alpha}\right) \frac{\sum_{s \in W} \epsilon(s) e^{\lambda^{\prime}(s H)}}{\prod_{\alpha>0} \lambda^{\prime}\left(H_{\alpha}\right) \prod_{\alpha>0} \alpha(H)} \frac{\prod_{\alpha>0} \alpha(H)}{\prod_{\alpha>0}\left(e^{\alpha(H) / 2}-e^{-\alpha(H) / 2}\right)}
$$

is a meromorphic function on $\mathfrak{h}_{t}$ and it is analytic everywhere on $(-1)^{1 / 2} \mathfrak{h}_{t_{0}}$ and also on a suitable neighbourhood of zero in $\mathfrak{h}_{\mathrm{t}}$. We know from [5, Part III, p. 71] that if $H \in \mathfrak{h}_{t}$ and $|t|$ is sufficiently small $(t \in C)$ then

$$
\Phi^{*}(\lambda, t H)=\sum_{m \geqq 0} \frac{t^{m}}{m !} \xi_{\lambda}\left(H^{m}\right)
$$

where $\xi_{\lambda}$ is the (infinitesimal) character of the algebra $\mathfrak{X}$ corresponding to the linear function $\lambda$ on $\mathfrak{h}$.

$\Lambda$ being any linear function on $\mathfrak{h}_{\mathfrak{p}}$, consider the integral $\int_{K} e^{\Lambda(H(x, u))} d u$ which occurs in Theorem 2. (Notice that $K=K^{*}$ in our case and therefore $\int_{K} d u=1$.) We shall now express this integral in terms of the function $\Phi^{*}$.

Consider the representation $\pi_{\Lambda}$ of $G$ on $L_{2}(K)$ given by

$$
\pi_{\Lambda}(x) f(u)=e^{-(\Lambda+2 \rho)\left(H\left(x^{-1}, u\right)\right)} f\left(u_{x^{-1}}\right) \quad\left(x \in G, u \in K, f \in L_{2}(K)\right.
$$

in the notation of $[6, \S 12]$. Let $\mathfrak{S}$ be the smallest closed subspace of $L_{2}(K)$ which is invariant under $\pi_{\Lambda}(G)$ and which contains the constant function 1. Then we have seen in [5, Part IV] that the representation $\pi$ of $G$ induced on $\mathfrak{S}$ is quasi-simple and its infinitesimal character( $\left.{ }^{12}\right)$ is $\chi_{\Lambda}$ where $\Lambda$ is to be extended to a linear function on $\mathfrak{h}$ by putting it equal to zero on $\mathfrak{h}_{\mathfrak{g}}$. Let $\psi_{0}$ denote the vector in $\mathfrak{S}$ corresponding to the constant function 1 . Then if we denote the scalar product of two elements in the usual way we get

$$
\left(\psi_{0}, \pi(x) \psi_{0}\right)=\int_{K} e^{\Lambda(H(x, u))} d u .
$$

On the other hand suppose $x=\exp t H_{0}$ where $H_{0} \in \mathfrak{h}_{\mathfrak{p}_{0}}$ and $t \in R$. Then since $\psi_{0}$ is well-behaved under $\pi$ (see Lemma 34 of [6]),

$$
\left(\psi_{0}, \pi\left(\exp t H_{0}\right) \psi_{0}\right)=\sum_{m \geqq 0} \frac{t^{m}}{m !}\left(\psi_{0}, \pi\left(H_{0}^{m}\right) \psi_{0}\right)
$$

provided $|t|$ is sufficiently small. Now put $i_{+}(X)=\left(X+(-1)^{1 / 2} i(X)\right) / 2$, $i_{-}(X)=\left(X-(-1)^{1 / 2} i(X)\right) / 2(X \in \mathfrak{t})$. Then $\mathfrak{t}_{+}=i_{+}(\mathfrak{f}), \mathfrak{t}_{-}=i_{-}(\mathfrak{f})$ are ideals in $\mathfrak{g}$ and $\mathfrak{g}$ is their direct sum. Let $\mathfrak{X}_{+}$and $\mathfrak{X}_{-}$be the subalgebras of $\mathfrak{B}$ generated by $\left(\mathfrak{t}_{+}, 1\right)$ and $\left(\mathfrak{t}_{-}, 1\right)$ respectively. Then if $a \in \mathfrak{X}_{+}$and $b \in \mathfrak{X}, a b-b a=0$.

(12) This is easily seen by the argument used in the proof of Lemma 48 of [5]. 
Choose $H_{0}^{*} \in \mathfrak{h}_{0}$ such that $H_{0}=i\left(H_{0}^{*}\right)$. Then

$$
H_{0}=i\left(H_{0}^{*}\right)=2(-1)^{1 / 2}\left[i_{-}\left(H_{0}^{*}\right)-H_{0}^{*}\right] \text {. }
$$

Moreover $H_{0}{ }^{*}$ and $i_{-}\left(H_{0}{ }^{*}\right)$ commute and $\pi(\mathfrak{f}) \psi_{0}=\{0\}$. Hence

$$
\pi\left(H_{0}^{m}\right) \phi_{0}=\left(2(-1)^{1 / 2}\right)^{m} \pi\left(H_{-}^{m}\right) \psi_{0}
$$

where $H_{-}=i_{-}\left(H_{0}^{*}\right)$. On the other hand if $X, Y \in \mathfrak{t}$,

$$
\left[X, i_{-}(Y)\right]=\left[i_{-}(X), i_{-}(Y)\right] \text {. }
$$

Hence $[X, z]=\left[i_{-}(X), z\right]\left(X \in \mathfrak{f}, z \in \mathfrak{X}_{-}\right)$. Therefore we get a representation $\nu$ of $\mathfrak{f}$ on $\mathfrak{X}_{-}$such that $\nu(X) z=[X, z]$ ( $\left.X \in \mathfrak{f}, z \in \mathfrak{X}_{-}\right)$. Obviously $\nu$ is quasi semisimple (see Lemma 10 of [6]). Therefore if $z$ is any element in $\mathfrak{X}_{-}$, it follows from Lemma 7 of $[6]$ that $z \equiv z_{0} \bmod \nu(\mathfrak{f}) \mathfrak{X}_{-}$where $z_{0}$ is some element of $\mathfrak{X}$ - which commutes with $\mathfrak{f}$. But then

$$
\left[i_{-}(X), z_{0}\right]=\left[X, z_{0}\right]=0
$$

and moreover $z_{0}$ commutes with $\mathfrak{f}_{+}$since it lies in $\mathfrak{X}_{-}$. Therefore $z_{0}$ is in the center of $\mathfrak{B}$. Furthermore the representation of $K$ induced under $\pi$ is unitary and therefore if $X \in \mathfrak{f}_{0}$ and $a \in \mathfrak{B}$,

$$
\left(\psi_{0}, \pi(X a-a X) \psi_{0}\right)=\left(-\pi(X) \psi_{0}, \pi(a) \psi_{0}\right)=0
$$

since $\pi(\mathfrak{f}) \psi_{0}=\{0\}$. This shows that

$$
\left(\psi_{0}, \pi(z) \psi_{0}\right)=\left(\psi_{0}, \pi\left(z_{0}\right) \psi_{0}\right)=\chi_{\Lambda}\left(z_{0}\right)
$$

Now if we extend $\chi_{\Lambda}$ to a linear function on $\mathfrak{B}$ such that $\chi_{\Lambda}(a b)=\chi_{\Lambda}(b a)$ $(a, b \in \mathfrak{B})$ (see Part III of [5]) it follows that $\chi_{\Lambda}\left(z_{0}\right)=\chi_{\Lambda}(z)$. Hence

$$
\left(\psi_{0}, \pi(z) \psi_{0}\right)=\chi_{\Lambda}(z)
$$

This proves that

$$
\left(\psi_{0}, \pi(\exp t H) \psi_{0}\right)=\sum_{m \geqq 0} \frac{t^{m}}{m !}\left(2(-1)^{1 / 2}\right)^{m} \chi_{\Delta}\left(H_{-}^{m}\right) .
$$

We extend $i_{-}$to an isomorphism of $\mathfrak{X}$ with $\mathfrak{X}_{-}$. Then the mapping $z \rightarrow \chi_{\Lambda}\left(i_{-}(z)\right)$ $(z \in \mathfrak{X})$ is clearly a character of the algebra $\mathfrak{X}$. Hence from Theorem 5 of [5] there exists a linear function $\lambda$ on $\mathfrak{h}_{\mathfrak{t}}$ such that $\chi_{\Lambda}\left(i_{-}(z)\right)=\xi_{\lambda}(z)(z \in \mathfrak{X})$. Therefore

$$
\left(\psi_{0}, \pi\left(\exp t H_{0}\right) \psi_{0}\right)=\sum_{m \geqq 0} \frac{t^{m}}{m !}\left(2(-1)^{1 / 2}\right)^{m} \xi_{\lambda}\left(H_{0}^{* m}\right)=\Phi^{*}\left(\lambda, 2(-1)^{1 / 2} t H_{0}^{*}\right)
$$

if $|t|$ is sufficiently small. In view of equation (25) (p. 81) of [5], $\lambda$ may be chosen in such a way that $\lambda(H)=\Lambda\left(i_{-}(H)\right)\left(H \in \mathfrak{h}_{\mathfrak{t}}\right)$. Since $\Lambda$ vanishes on $\mathfrak{h}_{\mathfrak{t}}$ we conclude that $\lambda(H)=-\left((-1)^{1 / 2} / 2\right) \Lambda(i(H))$ and therefore $2(-1)^{1 / 2} \lambda\left(H_{0}{ }^{*}\right)$ $=\Lambda\left(H_{0}\right)$. Put $s i(H)=i(s H) \quad(s \in W)$ and $\alpha(i(H))=(-1)^{1 / 2} \alpha(H), \sigma(i(H))$ 
$=(-1)^{1 / 2} \sigma(H)\left(H \in \mathfrak{h}_{\mathfrak{t}}\right)$. Then $\lambda\left(H_{\alpha}\right)=-\left((-1)^{1 / 2} / 2\right) \Lambda\left(i\left(H_{\alpha}\right)\right)=\Lambda\left(H_{\alpha}^{\prime}\right)$ where $H_{\alpha}^{\prime}=-\left((-1)^{1 / 2} / 2\right) i\left(H_{\alpha}\right)$ and $\sigma\left(H_{\alpha}^{\prime}\right)=\sigma\left(H_{\alpha}\right) / 2$. Then if we put

$$
\Phi(\Lambda, H)=\frac{\prod_{\alpha>0} 2 \sigma\left(H_{\alpha}^{\prime}\right)}{\prod_{\alpha>0}(\Lambda+2 \sigma)\left(H_{\alpha}^{\prime}\right)} \frac{\sum_{s \in W} \epsilon(s) e^{(\Lambda+2 \sigma)(s H)}}{\prod_{\alpha>0}\left(e^{\alpha(H)}-e^{-\alpha(H)}\right)} \quad\left(H \in \mathfrak{h}_{\mathfrak{p}_{0}}\right)
$$

it is clear that $\Phi(\Lambda, H)$ is an analytic function on $\mathfrak{h}_{\mathfrak{p}_{0}}$ and

$$
\Phi^{*}\left(\lambda, 2(-1)^{1 / 2} t H_{0}^{*}\right)=\Phi\left(\Lambda, t H_{0}\right) .
$$

Hence $\left(\psi_{0}, \pi\left(\exp t H_{0}\right) \psi_{0}\right)=\Phi\left(\Lambda, t H_{0}\right)$ for all sufficiently small values of $|t|$. Since both sides are analytic functions of $t$, the equality must hold for all values of $t$. Thus we have the following result.

Theorem 7. Let $\Lambda$ be a linear function on $\mathfrak{h}_{\mathfrak{p}}$. Then if $x=\exp H\left(H \in \mathfrak{h}_{\mathfrak{p}_{0}}\right)$ we have the formula

$$
\int_{K} e^{\Lambda(H(x, u))} d u=\frac{\prod_{\alpha>0} 2 \sigma\left(H_{\alpha}^{\prime}\right)}{\prod_{\alpha>0}(\Lambda+2 \sigma)\left(H_{\alpha}^{\prime}\right)} \frac{\sum_{s \in W} \epsilon(s) e^{(\Lambda+2 \sigma)(s H)}}{\prod_{\alpha>0}\left(e^{\alpha(H)}-e^{-\alpha(H)}\right)} .
$$

Put $\phi(x)=\int_{K} e^{\Delta(B(x, u))} d u=\left(\psi_{0}, \pi(x) \psi_{0}\right) \quad(x \in G)$. Then it is clear that $\phi(u x v)=\phi(x) \quad(u, v \in K)$. Since every element in $G$ can be written in the form $u(\exp H) v\left(H \in \mathfrak{h}_{\mathfrak{p}_{0}} ; u, v \in K\right)$, the above formula determines $\phi$ completely.

The particular case of this formula for the complex immodular group has been obtained by Gelfand and Naimark [2, p. 77] by means of a lengthy computation (see also [1]).

\section{REFERENCES}

1. Gelfand and Naimark, Doklady Nauk SSR (N.S.) vol. 63 (1948) pp. 225-228.

2. - Trudi Mat. Inst. Steklova vol. 36 (1950).

3. R. Godement, Trans. Amer. Math. Soc. vol. 73 (1952) pp. 496-556.

4. Harish-Chandra, Ann. of Math. vol. 51 (1950) pp. 299-330.

5. —-, Trans. Amer. Math. Soc. vol. 70 (1951) pp. 28-96.

6. - Trans. Amer. Math. Soc. vol. 74 (1953) pp. 185-243.

7. - Trans. Amer. Math. Soc. vol. 76 (1954) pp. 26-65.

8. - Proc. Nat. Acad. Sci. U.S.A. vol. 37 (1951) (a) pp. 170-173; (b) pp. 362-365;

(c) pp. 366-369; (d) pp. 691-694.

9. L. Schwartz, Theorie des distributions, Paris, Hermann, 1950.

10. I. E. Segal, Proc. Amer. Math. Soc. vol. 3 (1952) pp. 13-15.

Tata Institute of Fundamental Research, BOMBAY, INDIA.

Columbia University, NEW YoRK, N. Y. 